\title{
Das Ulcus adiposo-venosum - eine therapeutische Herausforderung
}

B. Kahle; A. L. Recke

Klinik für Dermatologie, Allergologie und Venerologie, Universität zu Lübeck, Lübeck

\section{Schlüsselwörter}

Adipositas, Ulcus cruris, chronische venöse Insuffizienz, Schaumsklerosierung

\section{Zusammenfassung}

Hintergrund: Die kausale Therapie eines UIcus cruris venosum stellt bei adipösen Patienten eine therapeutische Herausforderung dar. Methode: Anhand eines Fallberichts wird die Vorgehensweise demonstriert: mittels Sourcing kann auch bei schwerer Adipositas eine ulkusrelevante Varize detektiert und erfolgreich schaumsklerosiert werden.

Ergebnisse: Die erfolgreiche Schaumsklerosierung der im Sourcing relevanten Varize hat keinen Effekt auf die Ulkusheilung.

Schlussfolgerung: Stigmata der chronischen venösen Insuffizienz finden sich bei adipösen Patienten als Folge eben der Adipositas, so dass eine Gewichtsreduktion als kausale Therapie gelten kann. In unserem Fall kann von einem Ulcus adiposo-venosum gesprochen werden.
Keywords

Obesity, leg ulcers, chronic venous insufficiency, foam sclerotherapy

\section{Summary}

Background: The causal therapy of a venous leg ulcer is a therapeutic challenge in obese patients.

Method: Based on a case report, the procedure is demonstrated: Sourcing can be used to detect ulcer-relevant varices even in severe obesity and to successfully foam-sclerose them.

Results: The successful sclerotherapy of the sourcing-relevant varices has no effect on ulcer healing.

Conclusion: Stigmata of chronic venous insufficiency are found in obese patients as a result of obesity, so that weight loss can be considered a causal therapy. In our case, one can speak of an ulcer adiposo-venosum.

Korrespondenzadresse

Prof. Dr. med. Birgit Kahle

Klinik für Dermatologie, Allergologie und Venerologie Universität zu Lübeck

Ratzeburger Allee 160, 23538 Lübeck
Ulcer adiposo-venosum - a therapeutic challenge Phlebologie 2018; 47: 67-69

https://doi.org/10.12687/phleb2414-2-2018

Eingereicht: 05. Februar 2018

Angenommen: 18. Februar 2018

English version available at:

www.phlebologieonline.de

\section{Anamnese}

Eine 54-jährige Patientin wurde wegen eines therapierefraktären Ulcus cruris in die Klinik für Dermatologie, Venerologie und Allergologie des Universitätsklinikums Schleswig-Holstein, Campus Lübeck vorstellig. Seit mehr als 12 Jahren leidet die Patientin an einem rezidivierenden Ulcus cruris im Bereich des linken Innenknö- chels. Ebenfalls seit 12 Jahren hat die Patientin stetig an Gewicht zugenommen. Mittlerweile kann sie sich nur noch mühsam mit Hilfe eines Rollators fortbewegen. Die Patientin wird von einem häuslichen Pflegedienst versorgt und trägt konsequent Ulkuskompressionsstrümpfe. Darüber hinaus führt sie in ihrer häuslichen Umgebung regelmäßig eine intermittierende Kompressionsbehandlung durch.
Bei Vorstellung in der phlebologischen Abteilung zeigte sich am linken proximalen Unterschenkel eine massive Schwellung und distal eine ausgeprägte Dermatosklerose. Oberhalb des Malleolus externus bestand ein $7 \times 2 \times 1 \mathrm{~cm}$ messendes fibrinös belegtes Ulkus in sklerotischer Umgebung. ( Abb. 1). Die Fußpulse waren beidseits tastbar. Der Knöchel-Arm-Index war rechts 1,1 und links 1.06. Die Patientin hat bei einer Körpergröße von $1,82 \mathrm{~m}$ ein Gewicht von $220 \mathrm{~kg}$, was einem BMI >60 entspricht.

Die duplexsonographische venöse $\mathrm{Ab}$ klärung war in diesem Fall nur unter erheblich erschwerten Untersuchungsbedingungen möglich. Die Vena saphena magna und Vena saphena parva konnte beidseits sonographisch untersucht werden, sie waren jeweils kompetent. Die tiefen Venen ließen sich nur im Bereich der inguinalen und poplitealen Beugen darstellen, jeweils ohne Okklusion oder relevanten Reflux.

Aufgrund der enormen Schwellung der Beine bestanden im Wadenbereich eingeschränkte Schallbedingungen ( $\triangle$ Abb. 2). Dennoch konnte eine für das Ulkus hämodynamisch relevante Varikosis am Unterschenkel links detektiert werden. Durch das Sourcing-Phänomen $(1,2)$ konnte ein klarer Bezug dieser Varize zu der Ulzeration nachgewiesen werden.

\section{Relevante Begleiterkrankungen}

Es bestand eine diabetische Stoffwechsellage, eine arterielle Hypertonie und eine medikamentös eingestellte Depression.

\section{Verlauf}

Bezugnehmend auf den duplexsonographischen Befund wurde die Indikation zur Schaumsklerosierung der insuffizienten Unterschenkelvarizen links gestellt. 


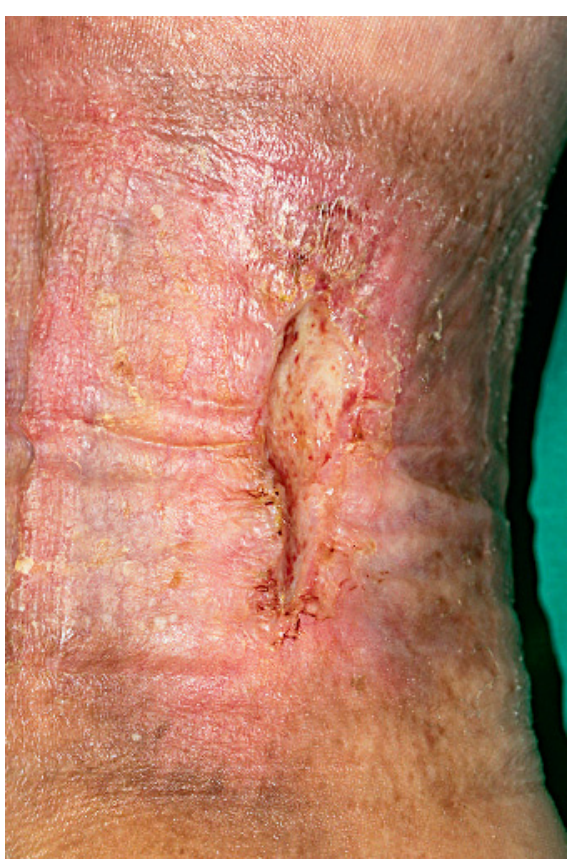

Abb. 1 Ulkus cruris am linken Außenknöchel in dermatoliposklerotischer Umgebung

Die Schaumsklerosierung selbst fand im Abstand von einer Woche in zwei Sitzungen komplikationslos statt. Verwendet wurde bei der ersten Sitzung 2\%iger Polidocanol-Schaum (Äthoxysklerol, Chemische Fabrik Kreussler, Wiesbaden), der in einer 1+4 Ratio mittels DSSTechnik (Double Syringe System) hergestellt wurde. In der zweiten Sitzung zeigte sich die Varize noch nicht komplett okkludiert, weshalb eine zweite Schaumsklerosierung nochmals mit 2\%igem Polidocanol durchgeführt wurde. Appliziert wurden jeweils $5 \mathrm{ml}$

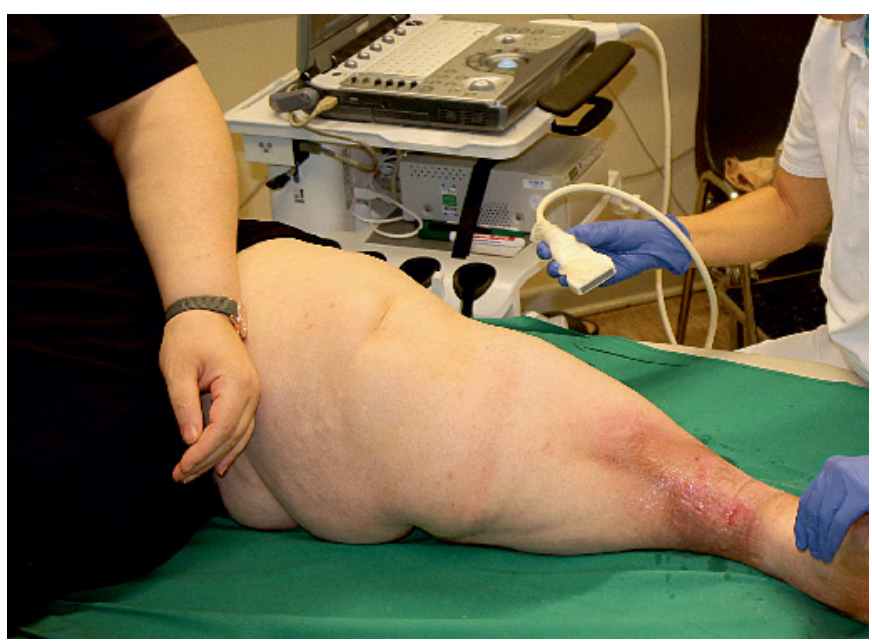

Abb. 2

Positionierung der $\mathrm{Pa}$ tientin zur Duplexsonographie, diese ist wegen der Ausmaße der Adipositas weder im Stehen noch im Liegen durchführbar zwischen Übergewichtigkeit und chronischer venöser Insuffizienz (CVI) besteht (4, 5). So zeigt die Bonner Venenstudie, dass das Risiko für das Auftreten einer CVI bei einem BMI > 30 signifikant erhöht ist - mit einer Odds ratio von 6,5 für Männer und 3,1 für Frauen (6). Eine weitere Studie zeigt eine positive Korrelation zwischen einem BMI $>30$ und dem Vorhandensein von Varizen bei postmenopausalen Frauen mit einer Odds ratio von 5,8 (7). Dagegen beschreiben andere Studien das Vorhandensein typischer Stigmata der CVI ohne pathologisches Korrelat in der venösen Strombahn bei Adipositas (8), auch schon $\mathrm{ab}$ einem BMI > 25 (9).

Im vorliegenden Fall war zunächst unklar, ob die bestehende, ausgesprochen therapierefraktäre Ulzeration in erster Linie als Folge der Adipositas gesehen werden muss, oder ob die im Sourcing relevante Varize eine Interpretation als venöses Ulkus in Folge einer chronischen venösen Insuffizienz rechtfertigt. Sicherlich sind hier beide Faktoren, die Adipositas und die Unterschenkelvarikosis, ätiologisch relevant. Da jedoch bei der Duplexsonographie die saphenofemorale Junktion sowie die saphenopopliteale Junktion und auch die $\mathrm{V}$ saphena magna beidseits suffizient waren, ist zu überlegen, ob hier nicht der Adipositas die Hauptrolle zukommt. Dennoch war hier das gezielte Ausschalten der Varikosis indiziert. Als Therapieoptionen waren eine gezielte Phlebektomie in Lokal- oder Tumeszenzlokalanästhesie oder eine sonographisch kontrollierte Schaumsklerosierung des relevanten Gefäßabschnittes zu erwägen. In Anbetracht der Adipositas sind operativen Verfahren gewisse Grenzen gesetzt, alleine die Lagerung, die Dauer des Eingriffs im Vergleich zur Schaumsklerosierung sowie das erhöhte Risiko von Wundheilungsstörungen sind dabei zu bedenken. Im Fall unserer Patientin kam aufgrund der Anatomie ihrer ausgeprägten Adipositas sowie der bestehenden Dermatosklerose die sonographisch kontrollierte Schaumsklerosierung als varizenausschaltende Maßnahme in Betracht. Aufgrund der adipösen Besonderheit im vorliegenden Fall konnte die Schaumsklerosierung nur im Sitzen ( $\triangleright$ Abb. 3) durchgeführt werden. Die Patientin konnte nicht flach liegen, da sie ansonsten erheblichste Luft- 
not bekam, und sie konnte auch nur ein Bein auf die Untersuchungsliege legen. Die Schaumsklerosierung erfolgte in zwei Sitzungen, die mit Sourcing detektierte relevante Varize konnte erfolgreich ausgeschaltet werden. Allerdings hatte dieses keinen signifikanten Effekt auf die Ulkusheilung. Da die Patientin verlässlich eine konsequente Kompressionstherapie durchführt, stellt sich die Frage, ob im vorliegenden Fall tatsächlich das Körpergewicht, im Speziellen das Übergewicht, die venöse Stauungssymptomatik begünstigt oder vielleicht sogar bedingt. Dafür spricht auch, dass nach der Schaumsklerosierung sonographisch keine varikösen Varizen mehr darstellbar waren. Die These, dass alleine eine abdominelle Druckerhöhung, wie sie bei einer Adipositas vorliegt, eine relevante venöse Stase und einen Anstieg des intravenösen Drucks in der Vena femoralis auslöst, konnte in einer in-vivo Studie eindrucksvoll gezeigt werden (10). Des Weiteren konnte dieselbe Arbeitsgruppe zeigen, dass die venöse Hämodynamik bei Patienten mit einem BMI > 30 ohne Zeichen einer CVI im Vergleich zu gesunden Probanden mit BMI <25 deutlich verändert ist (11).

Unser Fall veranschaulicht deutlich, dass eine schwere Adipositas Symptome einer CVI bedingen kann, ohne dass eine fassbare Inkompetenz der Venen in den betroffenen Extremitäten gesichert werden kann. In vielen Studien zeigt sich auch ein klarer Zusammenhang zwischen der Höhe des BMI und des C-Grades der CEAP Klassifikation $(12,13)$. Ferner konnte gezeigt werden, dass ein BMI > 30 mit einer gesteigerten Zunahme des zirkadianen Beinvolumens korreliert (14).

Zusammenfassend kann postuliert werden, dass Stigmata der CVI bis hin zum Ulcus cruris als Folge einer Adipositas auftreten können. Vielleicht sollte in solchen Fällen von einem Ulcus adiposo-venosum gesprochen werden.

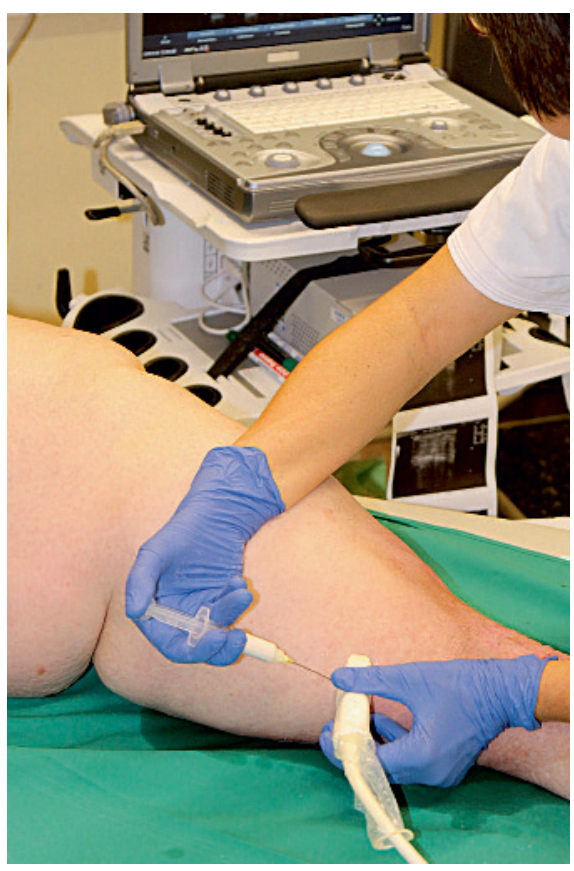

Abb. 3 Schaumsklerosierung der ulkusrelevanten Varize unter Ultraschallkontrolle

\section{Interessenkonflikt}

Die Autorin gibt an, dass kein Interessenkonflikt besteht.

\section{Ethische Richtlinien}

Die Arbeit wurde unter Einhaltung aller nationaler Richtlinien sowie der aktuellen Deklaration von Helsinki erstellt. Eine Einwilligung des Patienten liegt vor.

\section{Literatur}

1. Obermayer A, Garzon K. Sourcing of superficial reflux in venous leg ulcers using duplex ultrasound. J VAsc Surg 2010; 52: 1255-1261.

2. Niemer M, Miesel A, Kahle B. Sourcing - welche Varize ist ulkusrelevant? Phlebologie 2014; 43:180-182.
3. Mensink GBM, Schienkiewitz A, Haftenberger M, Lampert T, ZieseT, Scheidt-Nave C Ergebnisse der Studie zur Gesundheit Erwachsener in Deutschland (DEGS1), 2013 Abteilung für Epidemiologie und Gesundheitsmonitoring, Robert Koch Institut, Berlin, Bundesgesundheitsbl 2013; 56: 786-794.

4. Davies HO, Popplewell M, Singhal R, Smith N, Bradbury AW Obesity and lower limb venous disease - The epidemic of phlebesity. Phlebology. 2017 May;32(4):227-233. doi: 10.1177/0268355516649333. Epub 2016 May 13

5. Ageno W et al. Body mass index is associated with the development of the post-throm-botic syndrome. Thromb Haemost 2003; 89(2): 305-309.

6. Rabe E. Vein Bonn Study. Phlebologie 2006:179e86.

7. Iannuzzi A, Panico S, Ciardullo AV, Bellati C, Cioffi V, Iannuzzo G, et al. Varicose veins of the lower limbs and venous capacitance in postmenopausal women: relationship with obesity. J Vasc Surg 2002;36:965e8.

8. Padberg Jr F, Cerveira JJ, Lal BK, Pappas PJ, Varma S, Hobson RW. Does severe venous insufficiency have a different etiology in the morbidly obese? Is it venous? J Vasc Surg 2003;37:79e85

9. Danielsson G, Eklöf B, Grandinetti A, Kistner RL. The influence of obesity on chronic venous disease. Vasc Endovascular Surg 2002; 36: 271e6.

10. Willenberg T, Clemens R, Haegeli LM, AmannVesti B, Baumgartner I, Husmann M. The Influence of Abdominal Pressure on Lower Extremity Venous Pressure and Hemodynamics: A Human In-vivo Model Simulating the Effect of Abdominal Obesity Eur J Vasc Endovasc Surg 2011; 41: 849-855.

11. Willenberg T, Schumacher A, Amann-Vesti B, Jacovella V,Thalhammer C, Diehm N, Baumgartner I, Husmann M. Impact of obesity on venous hemodynamics of the lower limbs. J Vasc Surg 2010; 52: 664-668.

12. Van Rij AM, de Alwis CS, Jiang P, Christie RA, Hill GB, Dutton SJ, Thomson IA. Obesity and Impaired Venous Function, Eur J Vasc Endovasc Surg 2008; 35: 739-744.

13. Vlajinac HD, Marinkovic JM, Maksimovic MZ, Matic PA, Radak DJ. Body Mass Index and Primary Chronic Venous Disease - A Cross-sectional Study. Eur J Vasc Endovasc Surg 2013; 45: 293-298.

14. Engelberger RP, Indermühle A, Baumann F, Fahrni J, Diehm N, Kucher N, Egermann U, Laederach K, Baumgartner I, Willenberg T. Diurnal changes of lower leg volume in obese and non-obese subjects. Int J Obes 2014; 38: 801-805. 